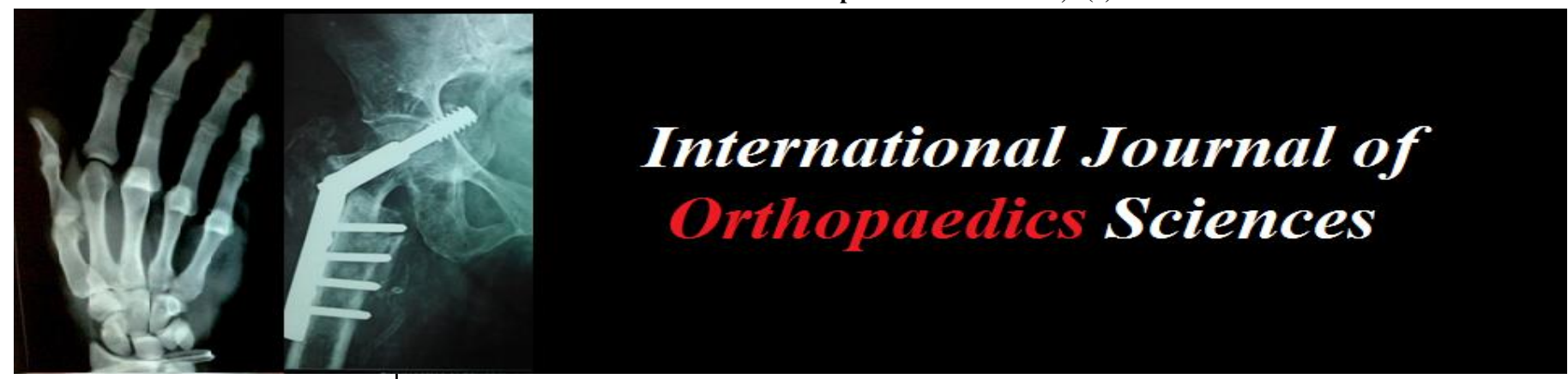

E-ISSN: 2395-1958

P-ISSN: 2706-6630

IJOS 2020; 6(2): 181-185

(C) $2020 \mathrm{IJOS}$

www.orthopaper.com

Received: 14-01-2020

Accepted: 18-02-2020

\section{Anant Akash}

Post Graduate, Department of

Orthopaedics, Sri Ram Murti

Smarak Institute of Medical

Sciences, Bareilly, Uttar

Pradesh, India

\section{Utkal Gupta}

Professor, Department of

Orthopaedics, Sri Ram Murti

Smarak Institute of Medical

Sciences, Bareilly, Uttar

Pradesh, India
Corresponding Author: Anant Akash

Post Graduate, Department of Orthopaedics, Sri Ram Murti

Smarak Institute of Medical

Sciences, Bareilly, Uttar

Pradesh, India

\section{A comparative analysis of the efficacy of intra-articular injections of corticosteroid, hyaluronic acid and platelet- rich plasma for the treatment of osteoarthritis knee}

\author{
Anant Akash and Utkal Gupta
}

DOI: https://doi.org/10.22271/ortho.2020.v6.i2c.2034

\section{Abstract}

Background: Osteoarthritis (OA) of the knee is the most common chronic degenerative joint disease characterized by pain, stiffness, swelling and progressive functional limitation in elderly. Non-surgical management modalities like physical therapy, lifestyle modification and oral non-steroidal antiinflammatory drugs, are often ineffective or do not alleviate symptoms adequately. Intra-articular corticosteroid (CS) and hyaluronic acid (HA) injections have been used for long to alleviate the symptoms of knee OA. Recently platelet-rich plasma (PRP) therapy has also been tried widely. Very few researches involving a comparison of the three have been conducted.

The purpose of the present study was to evaluate the therapeutic effect, efficacy and safety of intraarticular corticosteroid, hyaluronic acid and platelet-rich plasma for knee osteoarthritis.

Methods: 90 patients (132 knees) with primary knee osteoarthritis were randomized to receive one injection of intra-articular corticosteroid, hyaluronic acid or platelet-rich plasma and were followed for 3 months. The two groups were compared as regards pain and functional improvement using the Western Ontario and McMaster University Osteoarthritis Index (WOMAC), Knee injury and Osteoarthritis Outcome Score (KOOS), and the visual analog pain scale (VAS), by assessing the patients three times.

Results: VAS scale showed that all three modalities were effective in pain reduction. Both WOMAC and KOOS score represented variably improved symptoms i.e. decrease in pain and stiffness along with improvement in daily activity after 3 months in all 3 groups.

Conclusion: As the degree of pain relief achieved by the respective modalities was almost similar, the most important difference between the three intervention groups was the duration of their effectiveness. HA is suggested to be superior in the duration of pain relief when compared to CS and PRP. We can propose that HA can be administered less often as compared to CS and PRP for knee joint OA. Therefore, it will be more convenient to use HA.

Keywords: Osteoarthritis, Knee, Intra-articular injection, Hyaluronic acid, Corticosteroids, Platelet-rich plasma

\section{Introduction}

Osteoarthritis of the knee is the most common slowly progressive chronic degenerative joint disease, characterized by varying degrees of loss of joint cartilage with local inflammation, usually affecting the elderly population ${ }^{[1]}$. There is cartilage damage combined with a significant reduction in the viscoelastic properties of the synovial fluid and the molecular weight and concentration of the naturally occurring hyaluronic acid in synovial fluid decreases. This loss of visscoelasticity decreases the lubrication between joint surfaces and erodes the articular surfaces and is the mechanism of origin of pain in osteoarthritis ${ }^{[2]}$. The patient presents with pain, swelling, stiffness, deformity, decreased range of motion and disability, which significantly affect the quality of life. The knee is the most common joint that is affected in the Indian population with OA and plays an important role in weight bearing and mobility ${ }^{[3]}$.

Treatment is aimed at reducing symptoms i.e. reducing pain and inflammation and maintaining performance and normal movement of the joints and slowing the progression of the disease. It includes holistic therapeutic modalities including non- pharmacological measures like patient education, physical therapy with exercises to maintain range of motion and strength, lifestyle modifications such as dieting and weight reduction, walking supports (canes/crutches), 
bracing, shoe and insole modification and local hot/cold fomentation ${ }^{[4]}$. Pharmacologic therapies can be in the form of oral drugs like analgesics, non-steroidal anti-inflammatory drugs (NSAIDs), opioids, and newer drugs that help in cartilage regeneration like glucosamine, and chondroitin sulfate. Topical use of NSAIDs and Capsaicin is also recommended. If orally administered drugs are ineffective or inadequate, Intra-articular injections (corticosteroids, visscosupplements, and blood-derived products) can be offered as another non-operative modality ${ }^{[5]}$.

When conservative measures fail, operative measures such as arthroscopic debridement, high tibial osteotomy to redistribute loading of the joint or total knee arthroplasty can be considered ${ }^{[4]}$. OA has now become a major public health problem and a burgeoning financial burden for the global economy ${ }^{[6]}$.

Intra-articular CS has been a long-standing treatment for OA because of its anti-inflammatory and immunosuppressive effects. By acting directly on nuclear steroid receptors and interrupting the inflammatory and immune cascade, CS reduces vascular permeability and inhibit accumulation of inflammatory cells, phagocytosis, and prevents the synthesis and secretion of inflammatory mediators and increases relative viscosity and concentration of $\mathrm{HA}$ in the knee ${ }^{[7,8,9]}$. HA is a natural, non-sulfated glycosaminoglycan and a component of synovial fluid that can bind to specific receptors, triggering cytokine release and stimulation of cell cycle proteins, and stimulating cell migration and proliferation ${ }^{[10,11]}$. It acts as a lubricant of the joint and elastic shock absorber during joint movements. There is an increased susceptibility of cartilage breakdown in patients with osteoarthritis as the concentration and molecular weight of HA gets reduced ${ }^{[12,13,14]}$. The mechanism of action of intraarticular HA injection has not been completely understood but exogenous HA is long acting and has integral roles in improving joint lubrication and synovial fluid viscosity. It inhibits proteoglycan degradation and helps in the synthesis of hyaluronan. It also has analgesic and anti-inflammatory effects $[15,16,17]$.

PRP is an autologous supra-physiologic concentration of platelets in a small volume of plasma, prepared by centrifugation of blood. PRP contains a 3- to 5-fold increase in platelets concentration and $1-$ to 25 -fold higher concentration of cytokines and growth factors that are capable of stimulating cellular growth, vascularization, proliferation, tissue regeneration, and collagen synthesis. ${ }^{[18]}$ The release of platelet-derived factors directly at the site of cartilage disease, particularly with interest to knee OA, may stimulate the natural regenerative signaling cascade and enhance the healing of tissue with further mediation of the antiinflammatory response ${ }^{[19]}$.

The aim of the present study was to evaluate and compare the efficacy of intra-articular corticosteroid injections with HA and PRP for the treatment of knee OA.

\section{Materials and Methods}

This prospective randomized study was conducted in the outpatient department of our tertiary care hospital after approval of the institutional ethics committee, from October 2018 to January 2020. 90 patients (132 knees) of either sex, between 48 and 80 years of age, suffering from primary knee OA with Kellgren and Lawrence (KL) Grade II or III on standing antero-posterior and lateral knee radiographs [20], with symptoms for more than 3 months according to the ACR clinical classification criteria ${ }^{[21]}$ and pain score of more than
$40 \mathrm{~mm}$ on $100-\mathrm{mm}$ visual analog scale (VAS) were included in this study. Written informed consent was obtained from all patients before treatment.

Patients with history of significant trauma to the affected knee, active infection or tumors around the knee, secondary OA; having diabetes mellitus, cardiovascular diseases, coagulopathies, immunosuppressive, collagen, or autoimmune disorders or having a prior history of knee injections, hemoglobin values of $<10 \mathrm{~g} / \mathrm{dl}$ or platelet values of $<150,000 / \mathrm{ml}$; those receiving treatment with anticoagulant or anti-platelet medications or systemic corticosteroids 10 days before injection or recent use of NSAIDs; and pregnant and breastfeeding females were excluded from the study ${ }^{[4]}$.

$20 \mathrm{ml}$ of venous blood was drawn from the antecubital vein using an $18 \mathrm{G}$ needle to avoid traumatizing platelets and was collected in a sterile tube containing $2 \mathrm{ml}$ of Sodium Citrate anticoagulant. Approximately $2 \mathrm{ml}$ of whole blood was separated for a complete blood count ${ }^{[4]}$. Centrifuge was precooled at 2500 RPM for 15 minutes. Then, the blood with anticoagulant was centrifuged twice: First at $2700 \mathrm{rpm}$ for 12 min to separate erythrocytes and then at $3200 \mathrm{rpm}$ for $6 \mathrm{~min}$ to concentrate platelets. The final product was $4-5 \mathrm{ml}$ of PRPcontaining leukocytes with platelet concentration of 3-5 times the average normal value. This PRP was prepared in our hospital laboratory and was provided as required in a sterile form.

In all, 90 patients were randomly divided into 3 equal groups by random allocation sequence using a random-numbers table. The first group included 30 patients who were treated with one intra-articular injection of $2 \mathrm{ml}$ of methylprednisolone acetate at $40 \mathrm{mg} / \mathrm{ml} \mathrm{mixed} \mathrm{with} 2 \mathrm{ml}$ of lignocaine. The second group included 30 patients who were treated with a single $6 \mathrm{ml}$ intra-articular injection of HA by brand Hytas One (Intas Pharmaceuticals Ltd, India) at a concentration of $8 \mathrm{mg} / \mathrm{ml}$. The third group included 30 patients who were treated with a single $5 \mathrm{ml}$ intra-articular injection of PRP prepared in our hospital.

Before the administration of each injection, knee effusions if present, were aspirated into a separated syringe; the same needle was left in place and the syringe prefilled with CS, HA or PRP was used for injection [21].

The injection was given at a site near the superolateral pole of patella in the suprapatellar pouch under aseptic conditions ${ }^{[4]}$, with knee kept in 15-20 degree flexion and the patient was advised to take 1 day of rest after injection and apply ice to the area if there were any signs of inflammation ${ }^{[21]}$.

The Western Ontario and McMaster University Osteoarthritis Index (WOMAC), The Knee Injury and Osteoarthritis Outcome Score (KOOS) and the $100-\mathrm{mm}$ visual analog pain scale were used to assess the response to treatment at various intervals. The WOMAC was used as a self-administered test consisting of a 24-item questionnaire divided into three subscales which measure pain (5 items, score range 0-20), stiffness ( 2 items, score range $0-8$ ), and physical function (17 items, score range $0-68)^{[22]}$. The three normalized subscale values were summed to provide the normalized WOMACtotal score in the range of 0 (best score) to 96 (worst score). With the use of WOMAC, a lower score represented a better outcome ${ }^{[23]}$.

The KOOS measures both short-term and long-term outcomes of knee injury. It contains a 42-item questionnaire divided into five subscales measuring Pain, Other Symptoms, Activities of daily living (ADL), Function in Sport and Recreation (Sport/Rec), and Knee-related Quality of Life (QOL). All items have five possible answers from 0 (best 
score) to 4 (worst score) on a Likert Scale. While calculating the final score (0-100), a higher score represented a better outcome ${ }^{[24]}$.

The VAS, which also was administrated by the patient, ranged from 0 to $100 \mathrm{~mm}$, with lower numbers representing less pain and higher numbers representing more pain ${ }^{[21]}$. All the three measurements were used at the time of enrollment in the study before any injection and then the VAS was measured again at one, two and 3 months follow-up while the WOMAC and the KOOS were measured at the end of three months. The measurements were recorded in a pro forma and tabulated.

\section{Results}

Among the 90 patients enrolled in our study, 58 (64\%) were male and $32(36 \%)$ were female with a mean age of 64.7 . Out of 90 patients, there were 48 unilateral knee cases and 42 bilateral knee cases. A patient with bilateral knee pain was considered as one patient. $28(31 \%)$ patients had OA on the right knee and $20(22 \%)$ on the left knee, and the remaining $42(47 \%)$ had bilateral knee involvement. The mean age of the patients in the corticosteroid group were $64.7 \pm 9.1$ years, in hyaluronic acid group were $65.5 \pm 9.1$ years and in plateletrich plasma group were $63.8 \pm 9.4$ years. Males constituted
$80 \%$ in corticosteroid group, $70 \%$ in hyaluronic acid group and $43 \%$ in platelet-rich plasma group.



Fig 1: Percentage wise distribution of involved limbs in the study groups.

Table 1: Sex wise distribution of patients in the study groups.

\begin{tabular}{|c|c|c|c|c|c|c|}
\hline \multirow{2}{*}{} & \multicolumn{2}{|c|}{ Corticosteroid } & \multicolumn{2}{c|}{ Hyaluronic acid } & \multicolumn{2}{c|}{ Platelet-rich plasma } \\
\cline { 2 - 7 } & Number & Percentage & Number & Percentage & Number & Percentage \\
\hline Male & 24 & 80 & 21 & 70 & 13 & 43 \\
\hline Female & 6 & 20 & 9 & 30 & 17 & 57 \\
\hline
\end{tabular}



Fig 2: Percentage distribution of male and female patients in the three study groups.

\section{Pain (VAS)}

The VAS score before intervention in corticosteroid group was $7.1 \pm 2.1$, in hyaluronic acid group was $7.2 \pm 1.9$ and in platelet-rich plasma group was $7.1 \pm 2.3$. In corticosteroid group, pain at end of first month significantly decreased to $4.6 \pm 1.9$. At the end of second month, pain increased to $5.2 \pm 2.1$ and at the end of third month, pain score increased to $5.9 \pm 1.8$. In hyaluronic acid group, at end of first month, pain significantly decreased to $6.2 \pm 1.9$. Unlike corticosteroid group, pain continued its decreasing trend to $5.6 \pm 1.8$ at end of second month, but at the end of third month, pain score increased to $5.9 \pm 2.1$. In platelet-rich plasma group, at end of first month, pain significantly decreased to $6.4 \pm 2.2$. Like in hyaluronic acid group, pain continued its decreasing trend to $5.8 \pm 1.9$ at end of second month. At the end of third month, pain score increased to $6.1 \pm 2.1$ but it was also significantly lower than primary pain score.

Table 2: Comparison of VAS scores between the study groups at pre-intervention and 1,2 and 3 months post-intervention.

\begin{tabular}{|c|c|c|c|c|}
\hline VAS Score & $\begin{array}{c}\text { Pre- } \\
\text { intervention }\end{array}$ & $\begin{array}{c}\text { At 1 } \\
\text { Month }\end{array}$ & $\begin{array}{c}\text { At 2 } \\
\text { Month }\end{array}$ & $\begin{array}{c}\text { At 3 } \\
\text { Month }\end{array}$ \\
\hline Corticosteroid & $7.1 \pm 2.1$ & $4.6 \pm 1.9$ & $5.2 \pm 2.1$ & $5.9 \pm 1.8$ \\
\hline Hyaluronic Acid & $7.2 \pm 1.9$ & $6.2 \pm 1.9$ & $5.6 \pm 1.8$ & $5.9 \pm 2.1$ \\
\hline Platelet-rich plasma & $7.1 \pm 2.3$ & $6.4 \pm 2.2$ & $5.8 \pm 1.9$ & $6.1 \pm 2.1$ \\
\hline
\end{tabular}

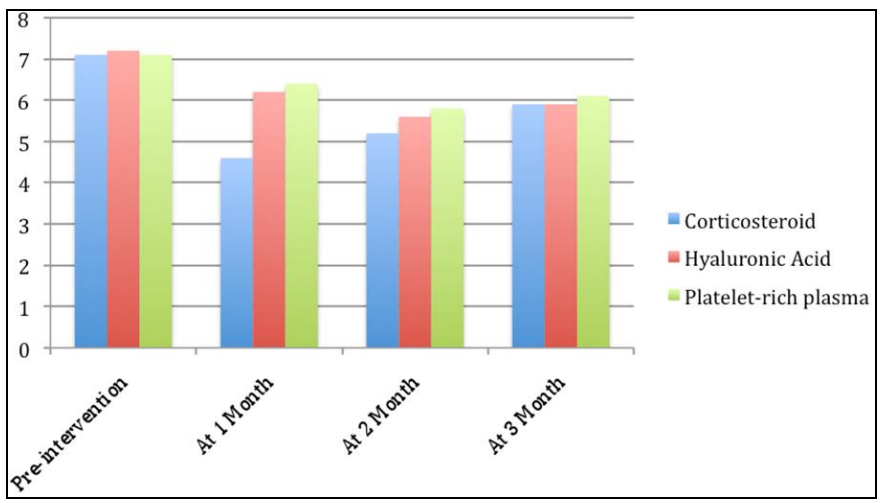

Fig 3: Comparison of VAS scores between the study groups at preintervention and 1, 2 and 3 months post-intervention.

\section{WOMAC score}

Pain and stiffness in all the three study groups decreased 3 months after intervention. Also physical function significantly improved in all three groups. The improvement was more significant in hyaluronic acid group as compared to both corticosteroid and platelet-rich plasma groups at the end of 3 months. 
Table 3: Comparison of WOMAC scores between the study groups.

\begin{tabular}{|c|c|c|c|c|c|c|}
\hline \multirow{2}{*}{ WOMAC score } & \multicolumn{2}{|c|}{ Corticosteroid } & \multicolumn{2}{c|}{ Hyaluronic acid } & \multicolumn{2}{c|}{ Platelet-rich plasma } \\
\cline { 2 - 7 } & Mean & SD & Mean & SD & Mean & SD \\
\hline Pain before (0-20) & 13.1 & 3.4 & 13.9 & 3.9 & 13.7 & 3.7 \\
\hline Pain after (0-20) & 12.7 & 3.5 & 13.2 & 3.8 & 13.1 & 3.6 \\
\hline Stiffness before (0-8) & 4.6 & 2.7 & 4.9 & 2.5 & 4.8 & 2.6 \\
\hline Stiffness after (0-8) & 4.4 & 2.6 & 4.3 & 2.4 & 4.4 & 2.6 \\
\hline Physical function before (0-68) & 37.8 & 12.1 & 38.1 & 12.2 & 37.9 & 12.1 \\
\hline Physical function after (0-68) & 33.2 & 11.9 & 32.9 & 12.1 & 33.1 & 12.1 \\
\hline
\end{tabular}

\section{KOOS Score}

Overall, all the parameters improved post-intervention after 3 months in all the three study groups. Improvement in pain after 3 months was less in both corticosteroid and platelet-rich plasma groups as compared to hyaluronic acid group. Daily activity also improved in all the study groups.

Table 4: Comparison of KOOS scores between the study groups.

\begin{tabular}{|c|c|c|c|c|c|c|}
\hline \multirow{2}{*}{ KOOS score } & \multicolumn{2}{|c|}{ Corticosteroid } & \multicolumn{2}{c|}{ Hyaluronic acid } & \multicolumn{2}{c|}{ Platelet-rich plasma } \\
\cline { 2 - 7 } & Mean & SD & Mean & SD & Mean & SD \\
\hline Pain before $(0-100)$ & 35.1 & 18.3 & 31.9 & 22.4 & 33.8 & 20.4 \\
\hline Pain after (0-100) & 38.1 & 19.3 & 34.8 & 22.5 & 36.6 & 20.6 \\
\hline Symptoms before $(0-100)$ & 39.8 & 18.7 & 40.4 & 22.3 & 40.1 & 21.1 \\
\hline Symptoms after (0-100) & 44.4 & 18.3 & 47.3 & 22.5 & 46.1 & 20.9 \\
\hline Daily activities Before (0-100) & 47.8 & 16.5 & 47.2 & 18.5 & 47.4 & 17.2 \\
\hline Daily activities After (0-100) & 52.2 & 16.1 & 52.3 & 18.7 & 50.5 & 17.4 \\
\hline
\end{tabular}

\section{Discussion}

Primary treatment goals in knee OA include pain reduction and improvement of joint mobility and function. The secondary goal is to decrease the progression of disease. Vissco-supplementation with hyaluronic acid is a non-operative intervention, which is frequently administered with the hope of achievement of both primary and secondary therapeutic goals over longer a longer duration as compared to corticosteroid, and platelet-rich plasma. ${ }^{[25]}$ These three categories of intra-articular injections need to be clinically evaluated comparatively to assign their indications, contraindications and to determine which treatment method was more effective for alleviation of pain and durability.

This study showed that in all the three study groups, the functions of the knee improved, but intra-articular HA injection were found superior to corticosteroid and plateletrich plasma in improving pain, stiffness, and functional status over a longer term.

VAS scale showed that all three modalities were effective in pain reduction at first, second and third month after intervention. The effectiveness of pain reduction was more durable in HA group as compared to CS and PRP. The pain score gradually increased at the second and third month endpoints after decreasing drastically at the first month endpoint but still remained better than pre-intervention levels. Both WOMAC and KOOS Scores showed improvement in all the parameters post-intervention after 3 months. The improvement was more significant in Hyaluronic acid group as compared to both corticosteroid and Platelet-rich plasma groups at the end of 3 months providing a good clue to compare the clinical efficacy of each intervention over 3 months.

Since the degree of pain relief was comparable across the study groups; consequently, it is argued that the most important difference between the three intervention modalities is the duration of effectiveness. Compared to CS and PRP groups, HA is suggested to be superior in the duration of pain relief. We can propose that intra-articular HA injections provide significant pain relief even after 3 months postintervention and the need for re-administration is less likely as compared to CS and PRP after every 3 months for knee joint OA.

There have been very few studies comparing the clinical outcomes of all three intra-articular injection modalities for OA knee. The superiority of our study as compared to others is in its reliance on several scales to clarify any differences between the three intra-articular injections. Unlike others, we did not focus only on the pain score and compared all the three modalities of intra-articular injections than comparing only two, which is the more common trend. Also none of the patients were lost during follow-up. We found that conducting studies with more extensive follow-ups and significantly larger sample size was demanding. In a prospective, randomized study, by Jones et al. ${ }^{[26]}$ patients withdrew during follow-up most commonly because of worsening of the symptoms over a longer term of conservative treatment thus challenging the utility of conservative methods of treatment of OA knee over a longer duration. ${ }^{[26]}$

\section{Conclusion}

According to results, the intra-articular HA injection was superior to both corticosteroid and platelet-rich plasma in pain relief and improvement of knee function over a period of 3 months. This supports the potential use of intra-articular HA as an effective long therapeutic option for patients with OA of the knee.

Financial support and sponsorship: Nil.

Conflicts of interest: There were no conflicts of interest.

\section{References}

1. Lawrence RC, Felson DT, Helmick CG, Arnold LM, Choi H, Deyo RA et al. Estimates of the prevalence of arthritis and other rheumatic conditions in the United States: Part II. Arthritis Rheum. 2008; 58(1):26-35.

2. Marshall KW. Viscosupplementation for osteoarthritis: Current status, unresolved issues, and future directions. J Rheumatol. 1998; 25:2056-8.

3. Jain P, Jain SK. Comparison of efficacy of 
methylprednisolone and triamcinolone in OA of the knee. Int J Sci Stud. 2015; 3:58-62.

4. Rai SK, Raman VP, Varma R, Wani SS. Combined intrarticular injections (Hyaluronic acid, platelet-rich plasma, and corticosteroid) for osteoarthritis knee, an effective alternate treatment. J Orthop Traumatol Rehabil. 2018; 10:57-60.

5. Kon E, Filardo G, Drobnic M, Madry H, Jelic M, van Dijk N, et al. Non-surgical management of early knee osteoarthritis. Knee Surg Sports Traumatol Arthrosc. 2012; 20:436-49.

6. Neogi T. The epidemiology and impact of pain in osteoarthritis. Osteoarthritis Cartilage. 2013; 21:1145-53.

7. Caldwell JR. Intra-articular corticosteroids: guide to selection and indications for use. Drugs. 1996; 52(4):507-514.

8. Ostergaard M, Halberg P. Intra-articular corticosteroids in arthritic disease: a guide to treatment. Bio Drugs. 1998; 9(2):95-103.

9. Creamer P. Intra-articular corticosteroid treatment in osteoarthritis. Curr Opin Rheumatol. 1999;11(5):417421.

10. Iannitti T, Lodi D, Palmieri B. Intra-articular injections for the treatment of osteoarthritis: focus on the clinical use of hyaluronic acid. Drugs RD. 2011; 11(1):13-27.

11. Cao JJ, Singleton PA, Majumdar S, Boudignon B, Burghardt A, Kurimoto P, Wronski TJ, Bourguignon LY, Halloran BP. Hyaluronan increases RANKL expression in bone marrow stromal cells through CD44. J Bone Miner Res. 2005; 20(1):30-40.

12. Dahl LB, Dahl IM, Engstrom-Laurent A, Granath K. Concentration and molecular weight of sodium hyaluronate in synovial fluid from patients with rheumatoid arthritis and other arthropathies. Ann Rheum Dis. 1985; 44(12):817-822.

13. Pelletier JP, Martel-Pelletier J. The pathophysiology of osteoarthritis and the implication of the use of hyaluronan and hylan as therapeutic agents in viscosupplementation. J Rheumatol Suppl. 1993; 39:1924.

14. Pelletier JP, Martel-Pelletier J, Raynauld JP. Most recent developments in strategies to reduce the progression of structural changes in osteoarthritis: today and tomorrow. Arthritis Res Ther. 2006; 8(2):206.

15. Vincent K. Hyaluronic acid (HA) viscosupplementation on synovial fluid inflammation in knee osteoarthritis: a pilot study. Open Orthop J. 2013; 7:378-384.

16. Goldberg VM, Buckwalter JA. Hyaluronans in the treatment of osteoarthritis of the knee: evidence for disease-modifying activity. Osteoarthritis Cartilage. 2005; 13(3):216-224.

17. Migliore A, Procopio S. Effectiveness and utility of hyaluronic acid in osteoarthritis. Clin Cases Miner Bone Metab. 2015; 12(1):31-33.

18. Drengk A, Zapf A, Stürmer EK, Stürmer KM, Frosch KH. Influence of platelet-rich plasma on chondrogenic differentiation and proliferation of chondrocytes and mesenchymal stem cells. Cells Tissues Organs. 2009; 189:317-26.

19. Mascarenhas R, Saltzman B, Fortier L, Cole B. Role of platelet- rich plasma in articular cartilage injury and disease. J Knee Surg. 2014; 28:003-010.

20. Kijowski R, Blankenbaker D, Stanton P, Fine J, De Smet A. Arthroscopic validation of radiographic grading scales of osteoarthritis of the tibiofemoral joint. AJR Am J
Roentgenol. 2006; 187:794-9.

21. Elsawy SA, Hamdy M, Ahmed MS. Intra-articular injection of hyaluronic acid for treatment of osteoarthritis knee: comparative study to intra-articular cotocisteroids. Egypt Rheumatol Rehabil. 2017; 44:143-6.

22. Nadrian H, Moghimi N, Nadrian E, Moradzadeh R, Bahmanpour K, Iranpour A, Bellamy N. Validity and reliability of the Persian versions of WOMAC Osteoarthritis Index and Lequesne Algofunctional Index. Clin Rheumatol. 2012; 31(7):1097-1102. doi: 10.1007/s10067-012-1983-7.

23. Insall JN, Dorr LD, Scott RD, Scott WN. Rationale of the knee society clinical rating system. Clin Orthop Relat Res. 1989; 248:13-14.

24. Roos EM, Lohmander LS. The Knee injury and Osteoarthritis Outcome Score (KOOS): from joint injury to osteoarthritis. Health Qual Life Outcomes. 2003; 1(1):64. doi: 10.1186/1477-7525-1-64.

25. Askari A, Gholami T, NaghiZadeh MM, Farjam M, Kouhpayeh SA, Shahabfard Z. Hyaluronic acid compared with corticosteroid injections for the treatment of osteoarthritis of the knee: a randomized control trail. Springerplus. 2016; 5:442.

26. Jones AC, Pattrick M, Doherty S, Doherty M. Intraarticular acid compared to intra-articular triamcinolone hexaacitonoide in inflammatory knee OA. Osteoarthritis Cartilage. 1995; 3:269-273. 